\title{
Funciones alométricas de contenido de carbono para quillay, peumo, espino y litre
}

\author{
Allometric functions of carbon content for quillay, peumo, espino and litre
}

\author{
Pablo Cruz ${ }^{\text {a*}}$, Alejandro Bascuñan ${ }^{\text {a }}$, Juan Velozo ${ }^{a}$, Marisol Rodrígueza

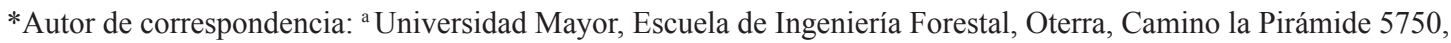 \\ Huechuraba, Santiago, Chile, tel: 02 23281367, tacora1@gmail.com
}

\begin{abstract}
SUMMARY
The National Forestry Corporation (CONAF) progresses in the implementation of the National Strategy for Climate Change and Vegetation Resources (ENCCRV). Under this context it is necessary to know the amount of carbon that can be contained by the Mediterranean Forest. The aim of this work was to development allometric functions to estimate carbon content in four species of the Chilean Mediterranean Forest: quillay, peumo, espino and litre. Nineteen sampling places, between Valparaíso and Biobío regions of the country, were selected for the cutting of 328 trees. Regarding the distribution of green weight between components, moisture content and basic density in espino and litre were similar, though different from quillay and peumo, which in turn showed similar levels to each other. According to these results, it could mean that there is influence of Mediterranean status over these variables. Carbon content in four species was around $49 \%$ of the total dry biomass. Models found provided similar settings to most of those already published for these species biomass, with the advantage that only one allometric variable is required for what is proposed in this work. Quillay, peumo and espino had $\mathrm{R}^{2}>0.92 \mathrm{while}$ espino had $\mathrm{R}^{2}=0.87$
\end{abstract}

Key words: allometric functions, carbon, forest mediterranean.

\section{RESUMEN}

La Corporación Nacional Forestal (CONAF) progresa en la ejecución de la Estrategia Nacional de Cambio Climático y Recursos Vegetacionales (ENCCRV), bajo este contexto es necesario conocer los contenidos de carbono que pueden contener el Bosque Mediterráneo. El objetivo de este trabajo fue la elaboración de funciones alométricas para estimar contenido de carbono en cuatro especies del Bosque Mediterráneo Chileno: quillay, peumo, espino y litre. Se abarcaron 19 zonas de muestreo distribuidas entre las regiones de Valparaíso y del Biobío con un total de 328 árboles procesados. Para la distribución de peso verde entre componente, para el contenido de humedad, y para la densidad básica, litre y espino fueron similares y diferentes a quillay y peumo, que a su vez mostraron niveles similares entre sí. Ello podría significar que existe influencia de la mediterraneidad en estas variables. El contenido de carbono para las cuatro especies oscila en torno a $49 \%$ de la biomasa seca total. Los modelos encontrados ofrecieron ajustes similares a la mayoría de los ya publicados para biomasa de las especies, con la ventaja que los propuestos en este trabajo, aportarán la componente carbono y sólo requieren una variable alométrica. Para quillay, peumo y espino el $\mathrm{R}^{2}$ encontrado fue mayor a 0,92 , mientras que para litre fue de 0,87 .

Palabras clave: funciones alométricas, carbono, bosque mediterráneo.

\section{INTRODUCCIÓN}

En Chile existe profusa información de funciones alométricas para biomasa de las especies arbóreas de los bosques templados (Schlegel 2001, Gayoso y Guerra 2005, Picard et al. 2012, Gayoso 2013abc.). Para las especies arbóreas del bosque mediterráneo, en cambio, los trabajos son más bien escasos. CONAF (2013) destaca la abundancia de publicaciones con funciones solo para especies de valor comercial, recopilando 21 funciones de biomasa para espino (Acacia caven Mol.), 28 para quillay (Quillaja saponaria Mol.), 26 para boldo (Peumus bodus Mol.), y solo tres para litre (Lithrea caustica Mol.) y ninguna para peumo (Cryptocarya alba (Mol.) Looser). Drake et al. (2003), en una recopilación anterior, encontraron 60 fun- ciones, tanto de biomasa y volumen, de las cuales el 80 $\%$ fue solo para el género Nothofagus. Así también, no se registran publicaciones científicas con funciones para estimación de carbono en estos bosques.

El objetivo de este trabajo fue la confección de nuevas funciones alométricas para el contenido de carbono aéreo para cuatro especies arbóreas del bosque mediterráneo chileno (quillay, peumo, espino y litre), y los atributos biométricos relacionados.

\section{MÉTODO}

Área de estudio. El estudio se desarrolló entre las regiones de Valparaíso y del Biobío, abarcando 19 zonas de muestreo cubriendo gran parte de la distribución geográ- 
fica de las cuatro especies (figura 1). Con colaboración de CONAF (Corporación Nacional Forestal) se ubicaron predios con planes de habilitaciones agrícolas y así evitar cortar árboles solo para este estudio.

Selección de árboles y medición de variables alométricas. Se seleccionaron 328 árboles, 80 quillay, 82 peumo, 80 litre y 86 espino en un periodo de doce meses. La distribución diamétrica de la muestra cubrió los rangos que muestra la figura 2 .

Una vez seleccionados, fueron cortados a ras de suelo y separados en los siguientes componentes:

- Tallo: sección leñosa entre el suelo y la clara generación de copa en varias bifurcaciones.

- Ramas: desde el tallo y hasta donde la sección transversal de la rama alcanza los $2 \mathrm{~cm}$.

- Ramillas y hojas: de las anteriores todo el resto.
A cada componente se le extrajeron tres probetas, las que fueron pesadas en terreno, empaquetadas y rotuladas para el procesamiento y análisis en laboratorio.

Las variables alométricas medidas en campo para la construcción de los modelos fueron diámetro a la altura del pecho (DAP, en $\mathrm{cm}$ ); diámetro altura de tocón (DAT, en $\mathrm{cm}$ ); altura total (HT, desde el suelo hasta la última rama viva, en metros); diámetro de copa (DC, dos diámetros, uno de norte a sur y otro de este a oeste, en metros).

En campo se observó que el espino generalmente forma un solo tallo en los primeros centímetros del suelo, ya sea de individuos de semilla o de rebrote. Aproximadamente a los $60 \mathrm{~cm}$ desde el suelo comienza a ramificarse en brotes secundarios (figura 3). Por esta razón, se tomó una variable adicional, correspondiente al diámetro de cada vástago después de la primera ramificación, regularmente un metro desde el suelo (diámetro a la altura del metro, DAM).

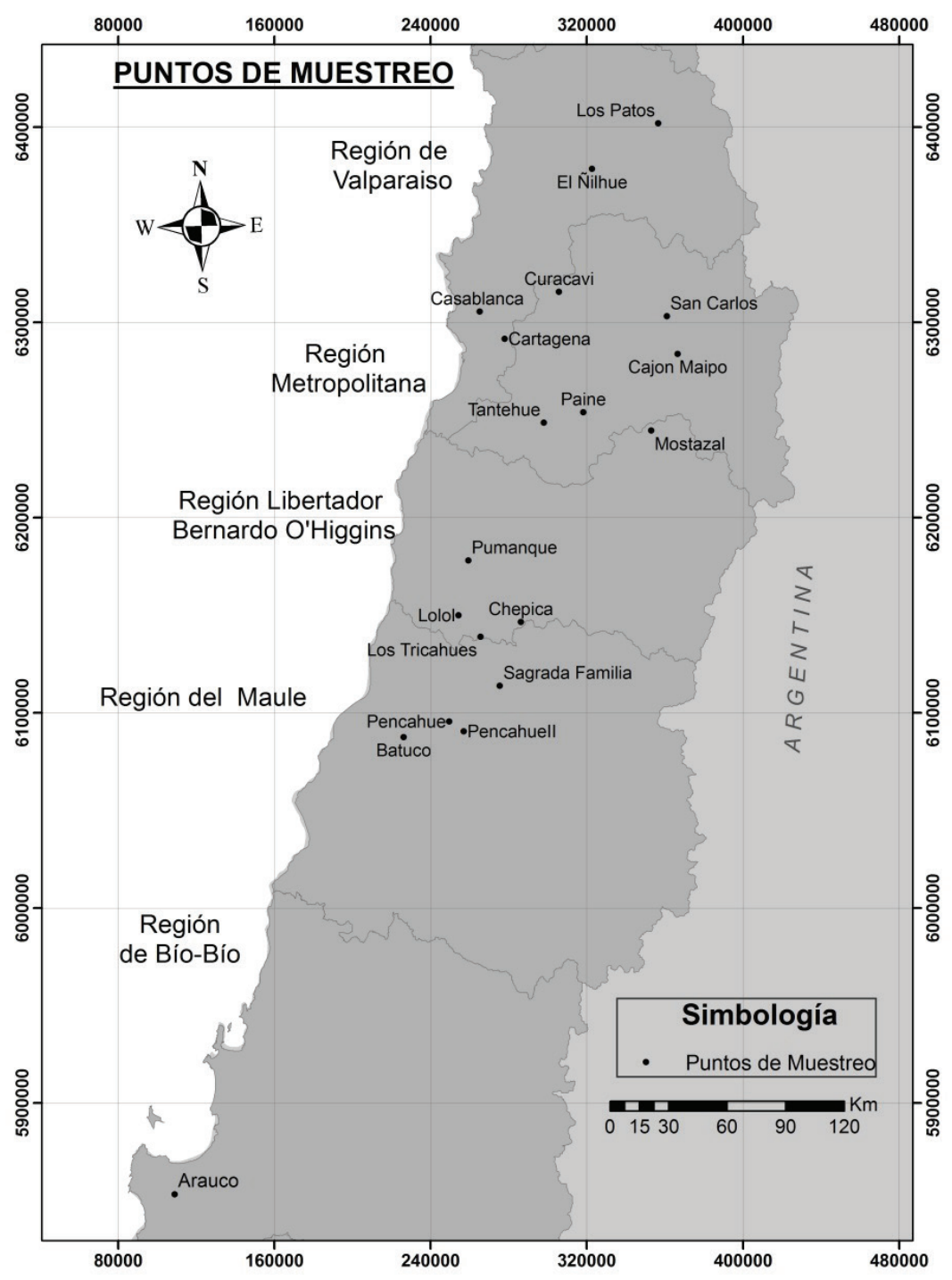

Figura 1. Ubicación geográfica de puntos de muestreo para las cuatro especies.

Geographic location of sampling points for the four species. 

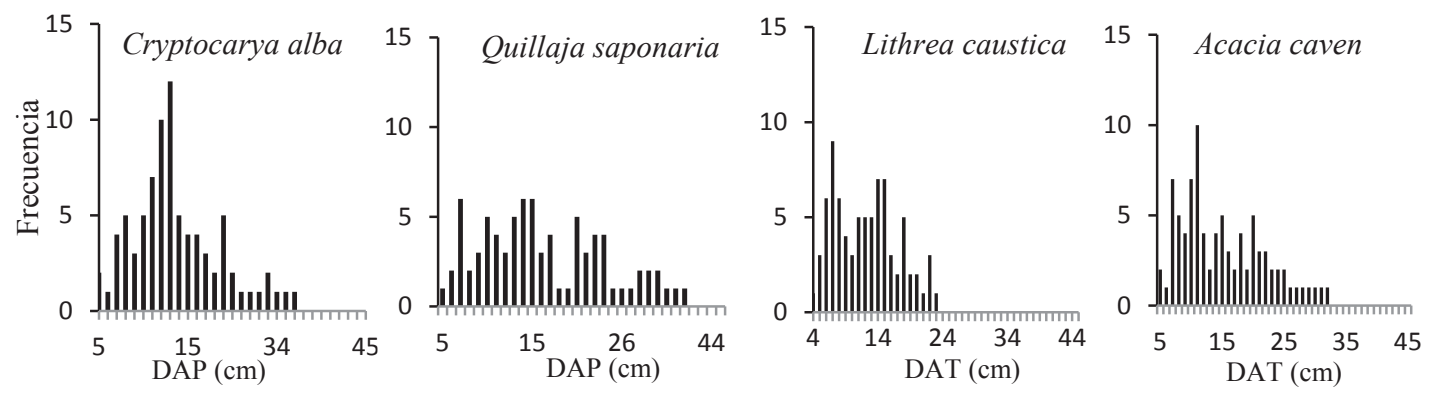

Figura 2. Distribución de frecuencias diamétricas.

Diametric distribution of frequencies.

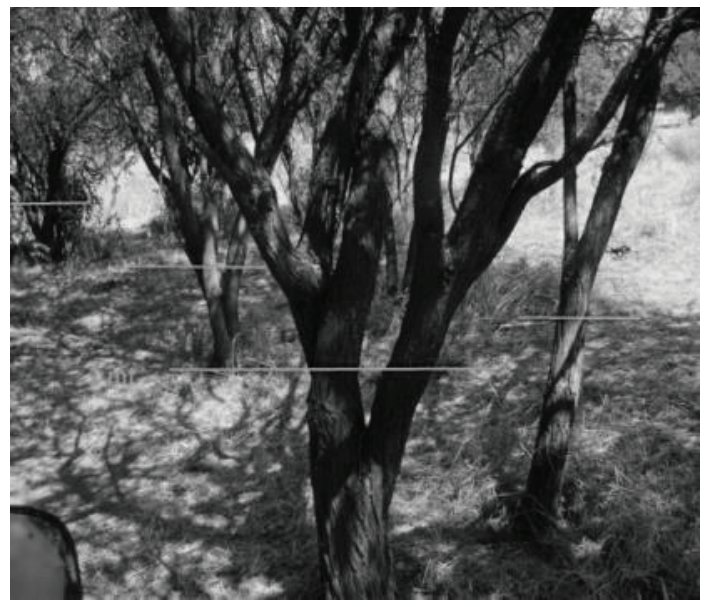

Figura 3. Comportamiento del crecimiento de vástagos.

Growth behavior of offspring.

El peso del tallo inferior se asignó proporcionalmente a cada vástago en función del diámetro relativo de cada uno.

Determinación variables biométricas. Para la determinación del contenido de humedad en base peso seco, se utilizó la norma chilena NCH 176/1-Of1984. Se calculó el contenido de humedad por el promedio de las tres repeticiones de cada componente. El peso fresco fue obtenido en terreno con una balanza granataria digital portátil, con una sensibilidad de $0,01 \mathrm{~g}$. La masa seca de las muestras fue obtenida por medio de secado en una estufa de tiro forzado a $105^{\circ} \mathrm{C}$; las muestras se mantuvieron por 72 horas hasta alcanzar peso constante.

La densidad básica de la madera se calculó según la norma chilena NCH176/2-1986. Se usaron probetas saturadas, y procedimientos para determinar la densidad de probetas irregulares. La estimación del volumen se realizó mediante la inmersión de las probetas y la medición del desplazamiento volumétrico por diferencia de pesadas. Las densidades se determinaron sobre probetas en forma de cuña, las que comprendieron desde el duramen hasta la albura.

El contenido de carbono se midió con el método Dumas con un equipo TruSpec CN, Leco®. Se tomaron muestras representativas de cada componente, para cada clase diamétrica y especie. De cada muestra se tomó una sub-muestra de $140 \mathrm{mg}$ de masa seca, la que fue molida homogéneamente. Para determinar el contenido de carbono se utilizaron estándares trazables NIST.

Para evaluar las diferencias entre componentes de las variables biométricas, se comprobó supuesto de normalidad mediante Kolmogorov-Smirnov y se realizaron análisis de varianza y posteriores análisis de contrastes múltiples (pruebas Duncan), cuando correspondió. Las diferencias entre especie para contenido de humedad $(\mathrm{CH})$ y densidad básica se hicieron con el mismo tipo de análisis, pero sólo para el total, mientras que el contenido de carbono, sólo para tallo.

Se cumplieron los supuestos de normalidad, lo que permitió aplicar análisis de varianza paramétrico y contrastes múltiples para cada variable.

Ajustes y selección de modelos. Se probaron once modelos de dos variables - dependiente y una independiente - y que generalmente tienen buenos ajustes para funciones de biomasa (Prodan et al. 1977) (cuadro 1). La selección del modelo se realizó según el coeficiente de determinación. Para litre sólo se usó diámetro de tocón, debido a que su hábito de crecimiento impide medir el resto de variables.

\section{RESULTADOS}

Distribución de la biomasa verde. La biomasa verde en el tallo es similar entre quillay y peumo, y considerablemente mayor que en litre y espino (cuadro 2).

Contenido de humedad. Se encontraron diferencias estadísticas del contenido de humedad entre los componentes en todas las especies $(P \leq 0,05)$ (cuadro 3 ). La componente hoja y ramilla es la más variable en todas las especies, debiéndose posiblemente a que su composición es intrínsecamente heterogénea, al tratarse de una mezcla de ramillas y hojas, éstas también de tamaño variable, siendo extremo en el caso de espino, con un cv de $51 \%$. Es posible que 
Cuadro 1. Modelos propuestos para estimar biomasa en especies del bosque mediterráneo chileno.

Models proposed to estimate biomass in Mediterranean species of the Chilean Forest.

\begin{tabular}{lc}
\hline \multicolumn{2}{c}{ Modelos simples de dos variables } \\
\hline$C T=\beta_{0}+\beta_{I} X$ & {$[1]$} \\
$C T=\beta_{0} \ln X+\beta_{1}$ & {$[2]$} \\
$C T=\beta 0+\beta 1(1 / x)$ & {$[3]$} \\
$C T=\beta_{0}+\beta_{I} X+\beta_{2} X^{2}$ & {$[4]$} \\
$C T=\beta_{0}+\beta_{I} X+\beta_{2} X^{2}+\beta_{3} X^{3}$ & {$[5]$} \\
$C T=\beta_{0}+\beta_{1}{ }^{x}$ & {$[6]$} \\
$C T=\beta_{0} X^{\beta 1}$ & {$[7]$} \\
$C T=\exp \left[\beta_{0}+\beta_{1}(1 / X)\right]$ & {$[8]$} \\
$C T=\exp \left[\beta_{0}+\beta_{I} X\right]$ & {$[9]$} \\
$C T=\beta_{0} \exp [\beta 1 X]$ & {$[10]$} \\
$C T=1 /\left(\beta_{0}+\beta_{1}+\beta_{2}{ }^{x}\right)$ & {$[11]$} \\
\hline Donde: & \\
$\mathrm{CT}=$ carbono total $[\mathrm{kg}]$ & \\
$\mathrm{X}=$ variable independiente & \\
$\mathrm{B}_{i}=$ parámetros a establecer para cada especie.
\end{tabular}

la variación en el espino se deba al ciclo fenológico de la hoja, considerando que la muestra se recogió en un período de doce meses.

Es destacable el alto nivel de contenido de humedad que presentó quillay, estadísticamente superior al resto en más de $12 \%$.

Densidad básica. Quillay y peumo no presentaron diferencias significativas entre componentes (cuadro 4). Espino y litre, en cambio, resultaron con densidades estadísticamente distintas entre componente $(P \leq 0,05)$. Así, también, se encontraron diferencias estadísticas entre todas las especies respecto de su densidad básica total.

Contenido de carbono. Es destacable el nivel constante que presentó el contenido de carbono en todos los componentes en todas las especies, que oscila entre 48 y $51 \%$ (cuadro 5). Para todos los casos, el componente hoja y ramillas tiene estadísticamente mayores contenidos de carbono respecto del resto $(P \leq 0,05)$. El contenido de carbono en el tallo entre especies, arrojó diferencias estadísticas entre algunas de ellas, a pesar que las diferencias entre los niveles son muy pequeñas.

Cuadro 2. Relación porcentual de biomasa y coeficiente de variación por componente en cada especie, según el pesaje en terreno.

Percentage ratio of biomass and coefficient of variation for each species component, according to their weight on land.

\begin{tabular}{|c|c|c|c|c|c|c|c|c|c|c|c|c|}
\hline \multirow{2}{*}{ Componente } & \multicolumn{3}{|c|}{ Quillay } & \multicolumn{3}{|c|}{ Peumo } & \multicolumn{3}{|c|}{ Litre } & \multicolumn{3}{|c|}{ Espino } \\
\hline & Tallo & Ramas & $\begin{array}{l}\text { Hojas y } \\
\text { ramillas }\end{array}$ & Tallo & Ramas & $\begin{array}{l}\text { Hojas y } \\
\text { ramillas }\end{array}$ & Tallo & Ramas & $\begin{array}{l}\text { Hojas y } \\
\text { ramillas }\end{array}$ & Tallo & Ramas & $\begin{array}{l}\text { Hojas y } \\
\text { ramillas }\end{array}$ \\
\hline Peso (\%) & 66 & 13 & 21 & 68 & 12 & 20 & 49 & 26 & 25 & 51 & 22 & 27 \\
\hline C.V. $(\%)$ & 16 & 33 & 39 & 14 & 38 & 41 & 27 & 37 & 35 & 24 & 29 & 40 \\
\hline
\end{tabular}

Cuadro 3. Contenido de humedad según tipo de muestra para las cuatro especies. El orden de posición de las especies en el cuadro obedece a su aparición en las prueba Duncan.

Moisture content as sample type for all four species. The rank order of the species in the table due to its appearance in the Duncan test.

\begin{tabular}{|c|c|c|c|c|c|c|c|c|}
\hline \multirow{2}{*}{ Componente } & \multicolumn{4}{|c|}{ Contenido de humedad (\%) } & \multicolumn{4}{|c|}{ Coeficiente de variación (\%) } \\
\hline & Litre & Espino & Peumo & Quillay & Litre & Espino & Peumo & Quillay \\
\hline Hojas y ramillas & $102,7 * *$ & $97,7 * *$ & $106,8^{* *}$ & $105,6^{* *}$ & 22 & 51 & 22 & 19 \\
\hline Ramas & $63,6^{*}$ & $74,3^{*}$ & $73,9 *$ & $91,9 *$ & 12 & 19 & 25 & 14 \\
\hline Tallo & 59,9 & 63,6 & 70,2 & 89,6 & 14 & 18 & 19 & 21 \\
\hline Total & $69,4^{\mathrm{a}}$ & $73,3^{\mathrm{ab}}$ & $77,0^{\mathrm{bc}}$ & $92,6^{\mathrm{d}}$ & - & - & - & - \\
\hline
\end{tabular}

$* P \leq 0,05$, Prueba Duncan entre especies para el total de Contenido de Humedad señalada con letras (a, b, c y d).

Total: promedio ponderado por el porcentaje del peso que cada componente tiene sobre el peso total. 
Selección de variables y modelos. La variable con mayor potencia predictora para quillay y peumo fue el diámetro a la altura del pecho (DAP). Litre es una de las especies que impone las mayores dificultades para la construcción de modelos como los de este trabajo, dado que no es posible determinar variables alométricas convencionales, salvo el diámetro a la altura del tocón (DAT). En espino, si bien el DAT ofrece buen desempeño, resultó mejor la nueva variable diámetro a la altura de un metro (DAM) (mejor ajuste y menor heterocesaticidad), por lo que se consideró oportuno proponer ambos modelos (cuadro 6, figura 4).

Cada vástago de la muestra contiene la proporción de biomasa de su cepa, de manera que para usar el DAM en inventario, bastaría medir todos los vástagos de la correspondiente parcela, independiente de las cepas.

Cuadro 4. Densidad básica según tipo de muestra. El orden de posición de las especies en el cuadro obedece a su aparición en las prueba Duncan.

Basic density according to sample type. The rank order of the species in the table due to its appearance in the Duncan test.

\begin{tabular}{ccccccccc}
\hline \multirow{2}{*}{ Componente } & \multicolumn{3}{c}{ Densidad básica $\left(\mathrm{Mg} \mathrm{m}^{-3}\right)$} & \multicolumn{3}{c}{ Coeficiente de variación $(\%)$} \\
\cline { 2 - 8 } & Peumo & Quillay & Litre & Espino & Peumo & Quillay & Litre & Espino \\
\hline Ramas & 0,64 & 0,66 & 0,71 & 0,72 & 10 & 5 & 8 & 8 \\
Tallo & 0,63 & 0,64 & $0,74^{*}$ & $0,73^{*}$ & 9 & 7 & 5 & 7 \\
\hline Total & $0,59^{\mathrm{a}}$ & $0,63 \mathrm{~b}$ & $0,69^{\mathrm{c}}$ & $0,72^{\mathrm{d}}$ & - & - & - \\
\hline
\end{tabular}

* $P \leq 0,05$, Prueba Duncan entre especies para el total de Densidad básica señalada con letras ( $\mathrm{a}, \mathrm{b}, \mathrm{c}$ y d).

Total: promedio ponderado por el porcentaje del peso que cada componente tiene sobre el peso total.

Cuadro 5. Contenido de carbono por componente y por especie. El orden de posición de las especies en el cuadro obedece a su aparición en las prueba Duncan para la componente tallo. stem component.

Carbon content per component and per species. The rank order of the species in the table due to its appearance in the Duncan test for the

\begin{tabular}{ccccccccc}
\hline \multirow{2}{*}{ Componente } & \multicolumn{3}{c}{ Contenido de carbono $(\%)$} & \multicolumn{4}{c}{ Coeficiente de variación (\%) } \\
\cline { 2 - 9 } & Litre & Quillay & Peumo & Espino & Litre & Quillay & Peumo & Espino \\
\hline Hojas y ramillas & $51,0^{*}$ & $50,1^{*}$ & $52^{*}$ & $51,0^{*}$ & 2,7 & 1,9 & 1,6 & 2,1 \\
Ramas & 48,5 & 48,8 & 49,0 & 48,3 & 1,2 & 1,4 & 1,3 & 1,8 \\
Tallo & $48,3^{\mathrm{a}}$ & $48,5^{\mathrm{ab}}$ & $49,0^{\mathrm{bc}}$ & $49,2^{\mathrm{c}}$ & 1,8 & 2,2 & 1,3 & 1,6 \\
\hline Total & 49,1 & 49,3 & 49,5 & 49,7 & & & & \\
\hline
\end{tabular}

* $P \leq 0,05$, Prueba Duncan entre especies para el total de Contenido de Carbono en el tallo, señalada con letras (a, b, c y d).

Total: promedio ponderado por el porcentaje del peso que cada componente tiene sobre el peso total.

Cuadro 6. Modelos seleccionados para la estimación de contenido de carbono.

Selected for estimation of carbon models.

\begin{tabular}{llccccc}
\hline \multicolumn{1}{c}{ Especie } & \multicolumn{1}{c}{ Modelos seleccionados } & & $\mathrm{R}^{2}$ & Error estándar & $\mathrm{N}$ & Rango diamétrico $(\mathrm{cm})$ \\
\hline Quillay & $\mathrm{CT}=0,0736 * \mathrm{DAP}^{2,251}$ & {$[12]$} & 0,95 & 0,254 & 80 & $\mathrm{DAP}=5-41$ \\
Peumo & $\mathrm{CT}=0,0675 * \mathrm{DAP}^{2,352}$ & {$[13]$} & 0,95 & 0,236 & 82 & $\mathrm{DAP}=5-37$ \\
Litre & $\mathrm{CT}=0,09638 * \mathrm{DAT}^{2,0912}$ & {$[14]$} & 0,87 & 0,349 & 80 & $\mathrm{DAT}=4-23$ \\
Espino & $\mathrm{CT}=0,03495 * \mathrm{DAT}^{2,3949}$ & {$[15]$} & 0,92 & 0,329 & 86 & $\mathrm{DAT}=5-32$ \\
Espino & $\mathrm{CT}=0,0871 * \mathrm{DAM}^{2,1416}$ & {$[16]$} & 0,94 & 0,249 & 96 & $\mathrm{DAM}=5-25$ \\
\hline
\end{tabular}

CT (kg): carbono total, DAP $(\mathrm{cm})$ : diámetro altura del pecho, DAT $(\mathrm{cm})$ : diámetro altura de Tocón; DAM $(\mathrm{cm})$ : diámetro a la altura de un metro. 

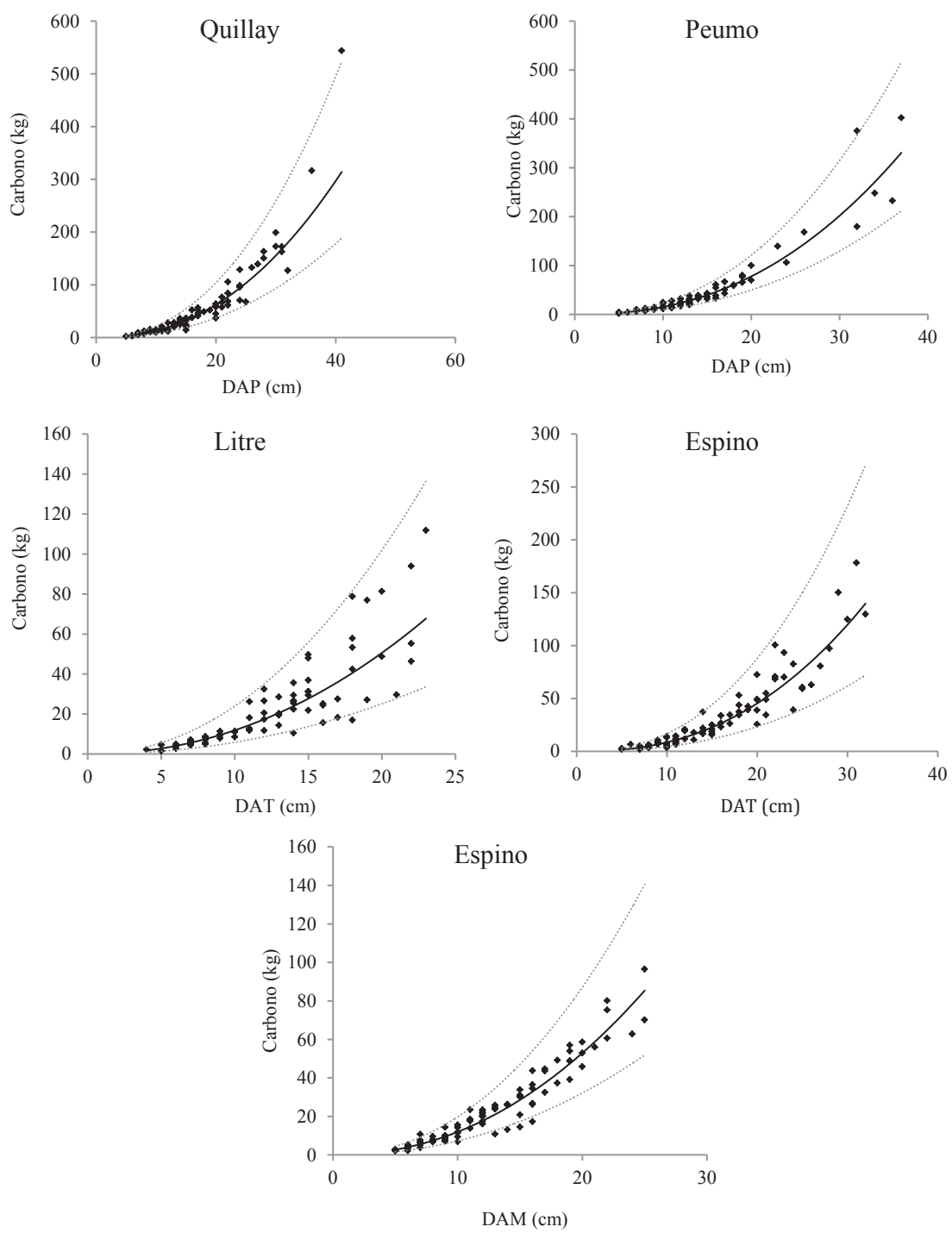

Figura 4. Modelos para la estimación de contenido de carbono para especies del bosque mediterráneo chileno.

Models for estimation of carbon content for the Chilean Mediterranean forest species.

\section{DISCUSIÓN Y CONCLUSIONES}

Contenidos de humedad. Gayoso et al. (2013abc) describen contenido de humedad con variación considerablemente mayor a las este estudio, para especies al sur de los bosque mediterráneos. Además, para Nothofagus obliqua (Mirb) Oerst., Nothofagus pumilio (Poepp. et Endl.) Krasser y Araucaria araucana (Molina) K. Koch estimaron contenidos de humedad medios de 97, 95 y $93 \%$, respectivamente, que comparado con los valores de peumo, litre y espino, permitiría afirmar que algunas de las especies del bosque mediterráneo mantiene menores contenidos de humedad que aquellas arbóreas de los bosques del sur del clima mediterráneo.

Contenidos de carbono. Las concentraciones de carbono encontradas son similares a las descritas para Nothofagus obliqua, Nothofagus pumilio y Araucaria araucana estudiadas por Gayoso et al. (2013abc). Gayoso y Guerra (2005) publican contenidos de carbono para 16 especies arbóreas de los bosques templados y siempreverdes, y para tallos sus estimaciones oscilan entre 42,6 y $47,7 \%$, que son inferiores a las encontradas en este estudio e incluso a las del mismo autor para especies similares. Las estimaciones de Yerena et al. (2012), para especies arbóreas y arbustivas en México también son significativamente menores (45,9\%). No obstante, trabajos de especies arbóreas en Europa y Asia coinciden con los valores en torno de $50 \%$ como los encontrados en este estudio. Joosten et al. (2004) determinaron concentraciones entre 49 y 50,7\% para Fagus sylvatica L. en bosques templados europeos, y Thomas y Malczewski (2007) estimaron concentraciones entre 49,5 y $50,8 \%$ y de 48,4 y $51,0 \%$ para bosques en Europa y China res- 
pectivamente. Thomas y Martin (2012), en su revisión de niveles de carbono para diversas especies según tipos de bosques, indican que en los bosques mediterráneos las especies tienen contenidos de carbono en un rango de $45 \mathrm{y}$ $60 \%$, y los resultados encontrados en este estudio reafirmarían esos resultados. Los autores mencionados advierten que existen grandes diferencias entre especies relativas al contenido de carbono, que no harían recomendable usar el valor por defecto de $50 \%$. No obstante, los resultados de este estudio indican que la variación en estas cuatro especies es baja, y si podría aceptarse un valor por defecto de $50 \%$ en bosques mediterráneos chilenos.

Tendencias entre las especies. Existe una cierta similitud entre litre y espino, y a su vez entre quillay y peumo en relación a sus proporciones de biomasa por componente, contenido de humedad y densidad básica. Así, pareciera ser que litre y espino distribuyeran más masa en su copa respecto de la mayor concentración en el tallo que hace quillay y peumo. Los contenidos de humedad en los tallos son menores en litre y espino, respecto de quillay y peumo, y su densidad básica es notoriamente mayor. Con estos resultados, se plantean dos interrogantes interesantes de analizar. En primer lugar, pareciera existir influencia de la mediterraneidad en las variables biométricas. De las especies estudiadas, espino se concentra en la zona norte del área, y litre tiende a establecerse en laderas de exposición norte, respecto de peumo y quillay. Para efectos prácticos de creación de líneas bases de contenidos de carbono en los bosques mediterráneos chilenos, sería interesante analizar la posibilidad de agrupar sus especies en función de los comportamientos de sus parámetros biométricos, considerando la diversidad de especies de este bosque.

En segundo lugar, las tendencias descritas no se verificaron para los contenidos de carbono, que si bien resultaron estadísticamente diferentes entre algunas especies, están en rangos estrechos entre ellas.

Modelos de carbono. La bondad de ajuste de los modelos encontrados para quillay y espino en este estudio son cercanos a los que CONAF (2013) recopiló de otros estudios a escala nacional. No obstante, aquellos modelos fueron construidos en todos los casos a partir de varias variables alométricas. Si bien modelos con múltiples variables permiten alcanzar ajusten de alta precisión, en muchos casos pueden quedar descartados debido al costo de ser aplicados a gran escala. Se destaca en este sentido la bondad de ajuste de los modelos encontrados y que sólo requieren una variable Alométrica. En este sentido, se destaca la nueva variable para espino (diámetro a la altura de un metro, DAM), por tener mejor ajuste que el DAT y más homocedasticidad.

\section{AGRADECIMIENTOS}

Este trabajo fue realizado usando las información del proyecto "Funciones alométricas para el bosque mediterráneo chileno", financiado por la Corporación Nacional Forestal en el marco de la Estrategia Nacional de Cambio Climático y Recursos Vegetacionales (ENCCRV) y el Fondo de Investigaciones Agrarias.

\section{REFERENCIAS}

CONAF (Corporación Nacional Forestal, CL). 2013. Compendio de funciones alométricas para la estimación de la biomasa de especies forestales presentes en Chile. Santiago, Chile. Corporación Nacional Forestal. 556 p.

Drake F, P Emanuelli, E Acuña. 2003. Compendio de funciones dendrométricas del Bosque Nativo. Santiago, Chile. CONAF y GTZ. 197 p.

Gayoso J, J Guerra. 2005. Contenido de carbono en la biomasa aérea de bosques nativos de Chile. Bosque 26(2): 33-38.

Gayoso J. 2013a. Funciones alométricas para la determinación de existencias de carbono forestal para la especie Araucaria araucana (Molina) K. Koch (araucaria). Santiago, Chile. CONAF. 49 p.

Gayoso J. 2013b. Funciones alométricas para la determinación de existencias de carbono forestal para la especie Nothofagus obliqua (Mirb.) Oerst. (roble). Santiago, Chile. CONAF. $41 \mathrm{p}$.

Gayoso J. 2013c. Funciones alométricas para la determinación de existencias de carbono forestal para la especie Nothofagus pumilio (Peopp. et Endl) Krasser (lenga). Santiago, Chile. CONAF. 32 p.

Joosten R, J Schumacher, C Wirth, A Schulte. 2004. Evaluating tree carbon predictions for beech (Fagus sylvatica L.) in western Germany. Forest Ecology and Management 189: 87-96.

Picard N, L Saint-André, M Henry. 2012. Manual de construcción de ecuaciones alométricas para estimar el volumen y la biomasa de los árboles. Rome, Italy. FAO. 213 p.

Prodan M, R Peters, F Cox, P Real. 1997. Mensura Forestal. San José, Costa Rica. IICA, GTZ. 561 p.

Schlegel B. 2001. Estimación de biomasa y carbono en bosques del tipo forestal siempreverde. In Simposio Internacional Medición y Monitoreo de la Captura de Carbono en Ecosistemas Forestales. Valdivia, Chile. 13 p.

Thomas S, A Martin. 2012. Carbon Content of Tree Tissues: A Synthesis. Forest 3(2): 332-352.

Thomas S, G Malczewski. 2007. Wood carbon content of tree species in Eastern China: Interspecific variability and the importance of the volatile fraction. Journal of Environmental Management. 85(3): 959-662.

Yerena J, J Jiménez, O Aguirre, E Treviño. 2012. Contenido de carbono total en los componentes de especies arbóreas y arbustivas en áreas con diferente uso, en el matorral espinoso tamaulipeco, en México. Bosque 33(2): 145-152. 
